\title{
List of reviewers 2019
}

We would like to thank those who contributed as peer reviewers in the period from January 2019 - December 2019 . Their anonymous work for the journal forms the foundation to its success and is highly appreciated.

Mohammed Abouh-Saleh

Shalini Ahuja

Mohammed Al-Uzri

Yaseen Algadal

Kamaldeep Bhui

Yvonne Bonomo

Nick Bouras

George Christodoulou

John Cox

Tom Craig

Julian Eaton

Mohamed Fakhr el Islam

Jane Fisher

Jorge Gaete

Alain Gregoire

Antoine Guedeney
Oye Gureje

Edvard Hauff

Dan Hayes

Carol Henshaw

Jonathan Herring

Louise Howard

Adrian James

Bhoomikumar Jegannathan

Rachel Jenkins

Jacob John

Elie Karam

Helen Killaspy

Christos Kouimtsidis

Marinos Kyriakopoulos

Petros Lekkos

Fadi Maalouf
Chin Yaet Mak

Sofia Manolesou

Daniel Maughan

Andrew Molodynski

Syed Asghar Naqi

Eleni Palazidou

Richard Rawson

Anne-Catherine Rolland

Khalid Saeed

Parag Shah

$\mathrm{Su}$ Tein Sim

David Skuse

Zebulon Taintor

Siri Tellier

Raymond Tempier 